\title{
Influence of Code Size Variation on the Performance of 2D Hybrid ZCC/MD in OCDMA System
}

\author{
Rima. Matem ${ }^{1, *}$, S. A. Aljunid ${ }^{1}, M . N$ Junita $^{1}$, C. B. $M$ Rashidi $^{1}$, Israa. Shihab Ahmed ${ }^{1}$ \\ Advanced Communication Engineering Centre of Excellence School of Computer and Communication Engineering \\ (ACE CoE -SCCE), Universiti Malaysia Perlis (UniMAP),Perlis, Malaysia,
}

\begin{abstract}
Several two dimensional OCDMA have been developed in order to overcome many problems in optical network, enhancing cardinality, suppress Multiple Access Interference (MAI) and mitigate Phase Induced Intensity Noise (PIIN). This paper propose a new 2D hybrid ZCC/MD code combining between 1D ZCC spectral encoding where $\mathrm{M}$ is its code length and 1D MD spatial spreading where $\mathrm{N}$ is its code length. The spatial spreading $(\mathrm{N})$ code length offers a good cardinality so it represents the main effect to enhance the performance of the system compared to the spectral (M) code length according to the numerical results.
\end{abstract}

\section{Introduction}

In OCDMA system, each user has its own code word rather of the specific wavelength or time slot which is provided by Wavelength Division Multiple Access (WDMA) and Time Division Multiple Access (TDMA) respectively [1]. This property provides that to users can access the same channel simultaneously and asynchronously [2]. Many 2D coding have been proposed and investigated as [3], [4], [5], [6]. Several classifications has been investigated in OCDMA system such as temporal OCDMA encoding and spectrumamplitude coding (SAC) OCDMA, spectral-phase encoding (SPE), frequency -hopping (FH) OCDMA, and 2D hybrid coding approach [7]. The 2D OCDMA depicts an enhanced performance compared to 1D OCDMA system in term of low power lost, high cardinality, minimum cross-correlation, high data rate and high autocorrelation with side[8] Several challenges affect the performance of OCDMA system in the implementation level [9-10]. The main challenges are due to the Multiple Access Interference (MAI) of the overlapping chips and Phase Induced Intensity Noise (PIIN) where PIIN is due to the desired signal mixed with the unwanted signal simultaneously at the same wavelength incident on the same photo-detector [11-12]. In the other side, an important factor can be impact the performance of OCDMA system is the code length where it represents one of the essential characteristic to design the system [13-14]. The main obstacle of increasing the code length is that growing complexity and cost, reducing the data transmission and bandwidth efficiency [15-16]. In this paper, combining between one dimensional Zero Cross Correlation code (1D ZCC) [17] and one dimensional Multi Diagonal code (1D MD) [18] where the both represent zero cross correlation property. The new 2D hybrid $\mathrm{ZCC} / \mathrm{MD}$ code used to eliminate MAI and mitigate PIIN. The increment of spatial code length $\mathrm{N}$ significantly influences the system performance compared to the spectral code length $M$. this paper is organized as follow: section II represents code construction, then section III shows the system performance, section IV depicts on the analytical results and discussion, finally the conclusion.

\section{2D hybrid ZCC/MD code construction}

The derivation of the $2 \mathrm{D}$ hybrid $\mathrm{ZCC} / \mathrm{MD}$ is based on the Zero Cross Correlation (1D ZCC) code and Multi Diagonal (1D MD) code. This new code is characterized by $\left(M, N, w, \lambda_{a}, \lambda_{c}\right)$ where $M$ is spectral code length, $N$ is spatial code length, $w$ is the code weight, $\lambda_{a}$ represents auto-correlation and $\lambda_{c}$ is the cross-correlation. The code size of the $2 \mathrm{D}$ hybrid code represented by $M \times N$.

\subsection{D Zero Cross Correlation (ZCC) code}

The Zero Cross Correlation (ZCC) code for SAC OCDMA was developed by [17] .ZCC is designed with zero cross correlation property between its code words to improve the performance of SAC OCDMA system. ZCC is characterized by a matrix of $K x L$ where $K$ represents number of active users and $L$ represents the minimum code length. The number of active user $K$ and code length $L$ are given as:

$$
\begin{aligned}
& K=w+1 \\
& L=w(w+1)
\end{aligned}
$$

The construction of the code is designed from the modified double weight (MDW) code [22] where the matrix is generated as follow with the basic ZCC code $(\mathrm{w}=1)$ :

$$
\operatorname{ZCC}(w=1)=\left[\begin{array}{ll}
0 & 1 \\
1 & 0
\end{array}\right]
$$

\footnotetext{
* Corresponding author: rymann90@hotmail.com
} 
According to the following maps, the increase of the number of users is occurred:

$$
\begin{aligned}
& \operatorname{ZCC}(w=2)=\left[\begin{array}{cc}
0 & Z_{1} \\
Z_{1} & 0
\end{array}\right] \\
& \operatorname{ZCC}(w=3)=\left[\begin{array}{cc}
0 & Z_{2} \\
Z_{2} & 0
\end{array}\right]
\end{aligned}
$$

The number of code weight can be increased by the general transformation below:

$$
Z C C_{w}=\left[\begin{array}{ll}
A & B \\
C & D
\end{array}\right]
$$

Where:

$A$ : is $[1, w(w-1)]$ matrix of zeros.

$B$ : represents $w$ replication of the matrix $\sum_{j=1}^{w} j[0,1]$.

$C$ : contain of the duplication of the matrix from $w-1$.

$D:$ is the diagonal pattern of $[m \times n]$ with exchange column of zeros matrix $[m \times n]$.

An example for the transformation code from $w=1 \rightarrow$ $w=2 \rightarrow w=3$ as follow:

$$
\begin{aligned}
& Z C C_{w=1}=-\frac{0}{1}: 1
\end{aligned}
$$

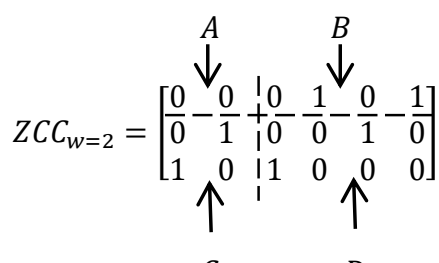

$$
\begin{aligned}
& \text { C D }
\end{aligned}
$$

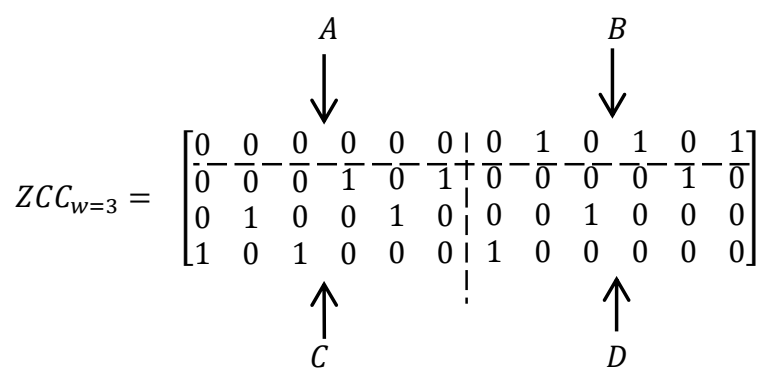

\subsection{D Multi Diagonal (MD) code}

One-dimensional Multi Diagonal (1D MD) was been developed by [19]. It is characterized by $N$ (code length), $w$ (code weight) and $\lambda_{c}$ (in phase cross correlation)

For the code sequence $X=\left\{x_{1}, x_{2}, \ldots, x_{N}\right\}, \quad Y=$ $\left\{y_{1}, y_{2}, \ldots, y_{N}\right\}$. The cross correlation expression can be expressed by $\lambda_{c}=\sum_{i}^{N} x_{i} y_{i}$

$1 D M D$ (Multi Diagonal) possesses zero cross correlation $\lambda_{c}=0$.

The matrix of 1D MD code consists of $K x N$ where $K$ is the number of user, $N$ is the code length. The choice of the weight value is free and $N=K w$.

The 1D MD code can be designed as below:

\section{Step1:}

A sequence of diagonal matrix can be constructed using the value of the weight $(w)$ and number of user $(K)$, according to these value the $i(i=1,2,3, \ldots . K)$ is the index of rows in each matrix and $j(j=$ $1,2,3, \ldots \ldots, w)$ where $\mathrm{j}$ is the number of diagonal matrix.

\section{Step2:}

The MD sequences can be computed for each diagonal matrix using the equation below:

$S_{i, j_{w}}=\left\{\begin{array}{c}\left(i_{n}+1-i\right), j_{w}=\text { even } \\ i \quad \text { for } j_{w}=\text { odd }\end{array}\right.$

$$
S_{i, 1}=\left[\begin{array}{c}
1 \\
2 \\
3 \\
\cdot \\
\cdot \\
\cdot
\end{array}\right], \quad S_{i, 1}=\left[\begin{array}{c}
K \\
\cdot \\
\cdot \\
\cdot \\
3 \\
2 \\
1
\end{array}\right], \quad S_{i, 1}=\left[\begin{array}{c}
1 \\
2 \\
3 \\
\cdot \\
\cdot \\
\cdot
\end{array}\right], \ldots
$$

Each element of the $S_{i, j}$ matrices represents the position of one in $T_{i, j}$ matrices with $K x K$ dimensions. Where

$$
\begin{gathered}
T_{i, 1}=\left[S_{i, 1}\right]_{K \times K}, T_{i, 2}=\left[S_{i, 1}\right]_{K \times K}, \cdots, T_{i, w}=\left[S_{i, 1}\right]_{K \times K} \\
T_{i, 1}=\left[\begin{array}{cccc}
1 & 0 & \cdots & 0 \\
0 & 1 & \cdots & 0 \\
\vdots & \vdots & \ddots & \vdots \\
0 & \cdots & \cdots & 1
\end{array}\right]_{K \times K}, T_{i, 2}=\left[\begin{array}{cccc}
0 & \cdots & 0 & 1 \\
0 & \cdots & 1 & 0 \\
\cdots & \cdots & \ddots & \vdots \\
1 & \cdots & \cdots & 0
\end{array}\right]_{K \times K}, \ldots, \\
T_{i, w}=\left[\begin{array}{cccc}
1 & 0 & \cdots & 0 \\
0 & 1 & \cdots & 0 \\
\vdots & \vdots & \ddots & \vdots \\
0 & \cdots & \cdots & 1
\end{array}\right]_{K x K}
\end{gathered}
$$

\section{Step3:}

\begin{tabular}{|c|c|}
\hline$i$ & Code sequence \\
\hline 1 & 1000000000110000 \\
\hline 2 & 01000001001000 \\
\hline 3 & 001001000010 \\
\hline 4 & 0001100000001 \\
\hline
\end{tabular}

The construction of the matrix of MD code of power $K x N$ is based on the combination of diagonals matrices. In a matrix each row is a single code sequence

$$
\begin{gathered}
M D=\left\lfloor T_{i, 1}: T_{i, 2}: \cdots \cdots\right. \\
M D=\left[\begin{array}{cccc}
a_{1,1} & a_{1,2} & \cdots & a_{1, N} \\
a_{2,1} & a_{2,2} & \cdots & a_{2,1} \\
a_{3,1} & a_{3,2} & \cdots & a_{3, N} \\
\vdots & \vdots & \cdots & \vdots \\
a_{i_{n}, 1} & a_{i_{n}, 2} & \cdots & a_{i_{n}, N}
\end{array}\right]
\end{gathered}
$$

The following table give an example of MD code with $K=4, w=3$

Table I: The MD code with $K=4, w=3$ 
TABLE II: 2D Hybrid ZCC/MD Code for $k_{1}=3$ and $k_{2}=2$

\begin{tabular}{|c|c|c|c|}
\hline$A_{g, h}$ & {$[101000100000]$} & {$[010010001000]$} & {$[000000010101]$} \\
\hline$\left[\begin{array}{l}1 \\
0 \\
0 \\
0 \\
0 \\
1\end{array}\right]$ & {$\left[\begin{array}{l}101000100000 \\
000000000000 \\
000000000000 \\
000000000000 \\
000000000000 \\
101000100000\end{array}\right]\left[\begin{array}{l}010010001000 \\
000000000000 \\
000000000000 \\
000000000000 \\
000000000000 \\
010010001000\end{array}\right]\left[\begin{array}{l}000000010101 \\
000000000000 \\
000000000000 \\
000000000000 \\
000000000000 \\
000000010101\end{array}\right]$} \\
\hline
\end{tabular}

The cross correlation of newly code can be obtained using four characteristics are given as follow:

$$
\begin{aligned}
& A^{0}=Y^{T} X \\
& A^{1}=Y^{T} \bar{X} \\
& A^{2}=\overline{Y^{T} X} \\
& A^{3}=\overline{Y^{T} X}
\end{aligned}
$$

Where $A(d), d \in(0,1,2,3)$ has been given in [19] represents the characteristic matrices. Where parameters $\bar{X}$ and $\bar{Y}$ are the complementary of $X$ and $Y$ respectively. The cross correlation of hybrid 2D ZCC/MD code $A^{(d)}$ and $A_{g, h}$ is formulated as:

$$
\left.R^{(d)}(g, h)=\right) \sum_{M=1}^{M-1} \sum_{N=1}^{N-1} a_{i j}^{(d)} a_{(i+g)(j+h)}
$$

Where $a_{i j}^{(d)}$ depicts the $(i, j)_{t h}$ of $A^{(d)}$ and $a_{(i+g)(j+h)}$ is the $(i, j)_{t h}$ of $A_{g, h}$. Table III shows the cross correlation between any two codes $A^{(d)}$ and $A_{g, h}$ of 2D Hybrid $\mathrm{ZCC} / \mathrm{MD}$ code produced from the equation (8).

Table III: Cross Correlation of 2D ZCC/MD hybrid code

\begin{tabular}{|c|c|c|c|c|}
\hline & $R^{(0)}$ & $R^{(1)}$ & $R^{(2)}$ & $R^{(3)}$ \\
\hline$g=0, h=0$ & $k_{1} k_{2}$ & 0 & 0 & 0 \\
\hline$g=0, h \neq 0$ & 0 & $k_{1} k_{2}$ & 0 & 0 \\
\hline$g \neq 0, h=0$ & 0 & 0 & $k_{1} k_{2}$ & 0 \\
\hline$g \neq 0, h \neq 0$ & 0 & 0 & 0 & $k_{1} k_{2}$ \\
\hline
\end{tabular}

The cross correlation of $A_{0,0}^{0}$ and $A_{g, h}$ can be calculated as follows:

$$
\begin{gathered}
R^{(0)}(g, h)=\sum_{i=1}^{M-1} \sum_{j=1}^{N-1} a_{i, j}^{(0)} a_{i, j}(g, h) \\
=\left\{\begin{array}{lc}
k_{1} k_{2} & \text { for } g=0, h=0 \\
0 & \text { otherwise }
\end{array}\right.
\end{gathered}
$$

In performance analysis there types of noise are considered Phase Induced Intensity Noise (PIIN), Shot Noise and Thermal Noise. In addition the BER is calculated using Gaussian approximation. The general form of the photocurrent noise from the photodiodes can be written as below:

$$
\begin{aligned}
\left\langle i_{\text {noise }}^{2}\right\rangle & =\left\langle i_{\text {PIIN }}^{2}\right\rangle+\left\langle i_{\text {shot }}^{2}\right\rangle+\left\langle i_{\text {thermal }}^{2}\right\rangle \\
& =B I^{2} \tau+2 e B I+\frac{4 K_{b} T_{n} B}{R_{L}}
\end{aligned}
$$

Where $B$ is the electrical bandwidth, $I$ is the average photocurrent, $e$ is the electron charge, $K_{b}$ is Boltzmann's constant, $T_{n}$ is the absolute temperature, $R_{L}$ is the load resistance and $\tau$ is the coherence time of the light. By applying the same method as [19], the PIIN, shot noise and thermal noise can be expressed as follow:

$$
\begin{aligned}
& \left\langle i_{\text {PIIN }}^{2}\right\rangle=B \frac{R^{2} P_{\text {Sr }}^{2} w_{2} k_{1}}{\Delta v W} \\
& \left\langle i_{\text {shot }}^{2}\right\rangle=2 e B \frac{R P_{s r} w_{2}}{W} \\
& \left\langle i_{\text {thermal }}^{2}\right\rangle=\frac{4 K_{b} T_{n} B}{R_{L}}
\end{aligned}
$$

Note that the probability of sending bit " 1 " at any time for each user is $1 / 2$ so:

$$
\left\langle i_{\text {noise }}^{2}\right\rangle=\frac{R^{2} P_{S r}^{2} w_{2} k_{1}}{2 \Delta v W}+\frac{e B R P_{s r} w_{2}}{W}+\frac{4 K_{b} T_{n} B}{R_{L}}
$$

where $R$ is the responsivity of the photo-diode given by $R=\eta e / h v_{0}, \eta$ is the quantum efficiency of the photo-diode, and $h$ is Plank's constant. $P_{s r}$ is the effective source power at the receiver, $k_{1}$ is the code weight of spectral encoding, $W$ is the number of active users, $N$ and $M$ are the code lengths of spatial and spectral code sequences respectively.

$S N R=\frac{I^{2}}{\left\langle i_{\text {noise }}^{2}\right\rangle}=\frac{\left[\frac{R P_{s r} w_{2}}{W}\right]^{2}}{\frac{R^{2} P_{s r}^{2} w_{2} k_{1}}{2 \Delta v W}+\frac{e B R P_{s r} w_{2}}{W}+\frac{4 K_{b} T_{n} B}{R_{L}}}$

The BER can then be calculated from SNR as follows [2]:

Where

$$
B E R=\frac{\operatorname{erfc}(\sqrt{S N R / 8})}{2}
$$

$$
\operatorname{erf} c=\frac{2}{\sqrt{\pi}} \int_{0}^{\infty} \exp \left(-y^{2}\right) d y
$$

\section{Results and Discussion}

Table IV: Parameters used in analytical calculations.

\begin{tabular}{|l|c|}
\hline $\begin{array}{l}\text { PD quantum } \\
\text { efficiency }\end{array}$ & $R=0.75$ \\
\hline $\begin{array}{l}\text { Spectral width of } \\
\text { broadband light } \\
\text { source }\end{array}$ & $\Delta \lambda=30 \mathrm{~nm}(\Delta \lambda=3.75 \mathrm{THZ})$ \\
\hline $\begin{array}{l}\text { Operating } \\
\text { wavelength }\end{array}$ & $\lambda_{0}=1.55 \mu \mathrm{m}$ \\
\hline $\begin{array}{l}\text { Electrical } \\
\text { bandwidth }\end{array}$ & $R_{b}=622 \mathrm{Mbps}$ \\
\hline $\begin{array}{l}\text { Data transmission } \\
\text { rate }\end{array}$ & $T_{n}=300 \mathrm{~K}$ \\
\hline $\begin{array}{l}\text { Receiver noise } \\
\text { temperature }\end{array}$ & $R_{L}=1030 \Omega$ \\
\hline $\begin{array}{l}\text { Receiver load } \\
\text { resistor }\end{array}$ & $K_{b}=1.38 \times 10^{-23} \mathrm{~W} / \mathrm{K} / \mathrm{Hz}$ \\
\hline $\begin{array}{l}\text { Boltzmann's } \\
\text { constant }\end{array}$ & $e=1.60217646 \times 10^{-19} \mathrm{coulombs}$ \\
\hline \begin{tabular}{l} 
Electron charge \\
\hline \begin{tabular}{l} 
Light velocity \\
\hline
\end{tabular}
\end{tabular} & $C=3 \times 10^{8} \mathrm{~m} / \mathrm{s}$ \\
\hline
\end{tabular}


The analysis of the code is based on the parameters shown in the Table IV where the numerical results are exhibited in following figures.

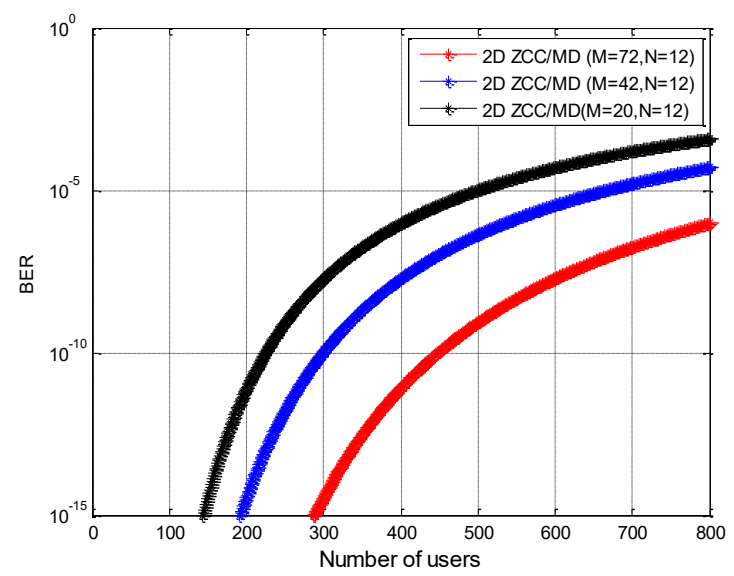

Figure 1: BER versus the number of simultaneous users for 2D $\mathrm{ZCC} / \mathrm{MD}$ with the fixed value number of time spreading $\mathrm{N}$

Figure1 illustrates the relation between the BER and the number of simultaneous users for $2 \mathrm{D} \mathrm{ZCC} / \mathrm{MD}$ with the fixed number of time spreading $\mathrm{N}$ to 12 and the effective source power, Psr is fixed at $-10 \mathrm{dBm}$ with variation of wavelength $M(M=20, M=42, M=72)$. It can be seen that this variation of $\mathrm{M}$ makes a small difference in the number of simultaneous users. The respective number of simultaneous users for groups of $(\mathrm{M}=20, \mathrm{~N}=12),(\mathrm{M}=42$, $\mathrm{N}=12)$ and $(\mathrm{M}=72, \mathrm{~N}=12)$ are 280,345 and 510 respectively when the BER at $10^{-9}$.

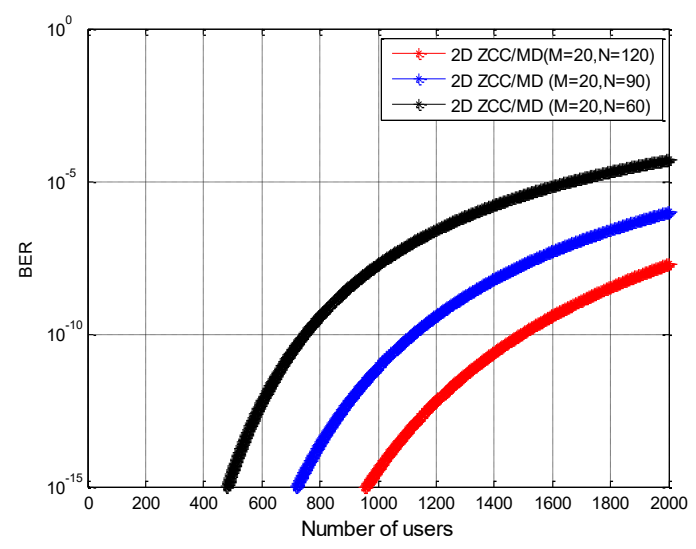

Figure2: BER versus the number of simultaneous users for 2D $\mathrm{ZCC} / \mathrm{MD}$ with the fixed number of wavelength $\mathrm{M}$.

The figure2 represents the plot of BER versus the number of users for $2 \mathrm{D} \mathrm{ZCC}$ with the fixed number of wavelength $\mathrm{M}$ to 20 and the time spreading $\mathrm{N}$ varies at 60,90 and 120 at Psr=-10 $\mathrm{dBm}$. According to the figure, the curves are separately each from other comparing with the Figure1. The changing of the values of $\mathrm{N}$ results the variation of cardinality. From the figure, at BER equal to $10^{-9}$ the number of simultaneous users for $\mathrm{N}=60,900$, 90 is 1350,120 and 1750 respectively. Note that the increases of $\mathrm{N}$ causes that the code can accommodate a higher number of simultaneous users but for the lowest number of $\mathrm{N}$ offer the lower cardinality in the system.

\section{Conclusion}

This study is focused to analyze 2D hybrid ZCC/MD code in term of impact size including 1D ZCC a spectral encoding and $1 \mathrm{D} \mathrm{MD}$ a spatial spreading. From the numerical results it can be seen that the improvement of the cardinality is related to the spatial spreading $\mathrm{N}$ where is present the principal role to ameliorate the performance of the system. The large number of $\mathrm{N}$ offers the capability to provide a high cardinality in the system at standard acceptable BER when the number of spectral encoding is fixe. The variation of $\mathrm{M}$ and fixed value of $\mathrm{N}$ makes a small difference in the number of simultaneous users can be obtained. This paper clarifies and defines the relationship between the code size and the system performance.

\section{References}

1. Paul R. Prucnal, CRC Press, Florida, (2006).

2. Keraf, N. Din, et al. Electronic Design (ICED), 2nd International Conference on. IEEE, (2014).

3. A.R Arief, S.A Aljunid, M.S Anuar, M.N Junita, R.B Ahmad and F. Ghani, IEEE International Conference on Control System, Computing and Engineering, pp. 488494, (2011)

4. Hongxi Y, Le Ma, Hongbin Li and Lixin Zhu, Photon New Communication, (2010)

5. Cheing-H L, Jingshown Wu and Chun-L Y. Journal of Lightwave Technology, vol. 23, no.12, pp. 3966-3980, December (2005)

6. Preeti $M$ and Shalini D. International Journal of Computer Science \& Engineering Technology IJCSET, pp. 1211-1215, (2012)

7. Arief, A. R., Aljunid, S. A., Anuar, M. S., Junita, M. N., \& Ahmad, R. B. Optik-International Journal for Light and Electron Optics, 124(19), 3786-3793. (2013)

8. Kadhim, R. A., Fadhil, H. A., Aljunid, S. A., \& Razalli, M. S. Journal of Theoretical and Applied Information Technology, 81(3), 589. (2015)

9. Abdullah, A. R. A. J., Aljunid, S. A., Safar, A. M., Nordin, J. M., \& Ahmad, R. B. Optical Engineering, 51(6), 065007-1. (2012)

10. Keraf, N. Din, et al. Photonics (ICP), 2014 IEEE 5th International Conference on. IEEE, 2014.

11. Chang, Yao-Tang, et al. Optical Fiber Technology 16.2: 114-123. (2010)

12. Keraf, N. Din, et al. Advanced Computer and Communication Engineering Technology. Springer, Cham. 129-140. (2015)

13. Keraf, N. Din. Malaysia University Conference Engineering Technology. (2014)

14. Jellali, Nabiha, et al. Optical Fiber Technology 36: 26-32. (2017)

15. Dexter, Karl J., Douglas Alexander Reid, and Liam P. Barry. IEEE photonics technology letters 21.22: 16621664. (2009) 
16. Keraf, N. Din, et al .Electronic Design (ICED), 2016 3rd International Conference on. IEEE, (2016).

17. M. S. Anuar, S. A. Aljunid, N. M. Saad \& S. M. Hamzah. Optics Communications, 282(14), 2659-2664. (2009).

18. A .Thanaa Hussein, S.A. Aljunid, H. Adnan, R.A. Ahmad, 2N.M. Saad. Australian Journal of Basic and Applied Sciences, 6(3): 112-119, (2012)

19. R.A. Kadhim, H. A. Fadhil, S. A. Aljunid \& M. S .Razalli. Optics Communications, 329, pp. 28-33. (2014). 